\title{
NUMERICAL SIMULATION OF A SMALL SIZE ROTOR WITH SELF- INDUCED INSTABILITY USING SLIDING MODE AND FOPID CONTROLLER AND THEIR COMPARISON
}

\author{
Abhro Mukherjee ${ }^{1}$, Satyabrata Das 2 \\ 1,2 National Institute of Science and Technology, Odisha \\ E-mail: abhro456@gmail.com, satyabrataresearch@gmail.com
}

\begin{abstract}
Dynamic systems like gyro-pendulums and tippe-tops behave in a peculiar unstable manner due to material or internal damping. Dynamic systems beyond certain critical threshold spinning speed may become unstable due to rotating dampers. There have been several efforts in the past to stabilize such systems but most of the earlier efforts to stabilize such rotors had difficulty in stabilizing extremely small size, mini or micro rotors although one may find some efforts to stabilize medium to large size rotors using some heavy actuators like squeeze film dampers etc. The underlying problems with the small size rotors is that if the selection of the actuators is not proper than the actuator size may be heavier than the rotor itself therefore the choice of actuators remains always a challenge in a rotor with micro or mini size.

Since in the present work the control, sensing and actuation all are embedded within a single embedded coupling due to obvious practical reasons as discussed in the paper the challenge is to see the entire dynamics from a moving frame or rotating coordinate system. Design and practical implementation of these smart structures always remains a big challenge.
\end{abstract}

Keywords: Internal Damping, Non-potential forces, Regenerative Power, Shaft drawn power, threshold spinning speed, fractional order PID controller.

\section{Introduction}

Industrial Rotors after a certain threshold or critical spinning speed may become completely unstable due the presence of internal damping or material damping rotating along with the rotor. There have been several efforts previously to stabilize large industrial rotors with heavy actuators but efforts to stabilize small size rotating shafts remained always a challenge and considered a major industrial problem specially for automation in semiconductor industry.

The paper also shows how the critical or threshold spinning speed of the rotor plays a pivotal role in designing the controller as because the control law has the tendency in general to stabilize the shaft by translating the threshold spinning speed way beyond the running speed of the rotor

After determination of the threshold critical spinning speed of the rotor one might get all the essential parameters on which the rotor critical speed actually depends upon and then based on practical implementation the manipulating variable is thus carefully chosen. The paper clearly shows that although the threshold speed is dependent on the rotor stiffness and external damping but clearly selecting the internal damping as the modulating parameter is more practical specially if one might use a smart embedded structure in stabilizing rotors in rotating frame. Finally, two different types of controller are designed. Firstly, a FOPID type of controller and then a well-tuned normal sliding mode controller. The comparison of the efficacy of each type of controller is done and the results discussed.

\section{Literature}

A wonderful paper describing 'Effects of biphase lubricants on Dynamics of rigid rotor' ASME [6] gave us an insight of physical dynamics of rotors in general and the effects of non-conservative type forces due to typical behavior of these biphase lubricants and its destabilization effects on the rotors dynamics. The paper talked a lot about the nature and effects of these non-potential forces on the rotor and the need for an stabilization technique.

In this paper no control issues as such was discussed and moreover the results shown in the paper was particularly for large size rotors.

A paper, "An Investigation in to the Physics Behind the Stabilizing Effect of Two Phase Lubricants in Journal Bearings" Journal of vibration and control [18] discussed almost the same issues of as in [6] but more in details regarding the 
instabilities in the journal bearing due to antisymmetric stiffness coefficients and general nature of bearing forces in the bearing system without much emphasizing on the control strategies to stabilize such systems. Paper [19] Kirillov O. N "Gyroscopic Stabilization in the Presence of Nonconservative forces" Dokl. Math, 2007, also dealt with the techniques to mechanically stabilize systems affected by non-conservative forces with heavy dampers once again not really suitable for rotors of small size and description. The instability issues of Tippe top dynamics because of internal damping of the rotor system was given in the paper [21] "Dissipation-Induced Heteroclinic Orbits in Tippe Tops" 2008, SIAM Review. The dynamics of gyro pendulum shows exactly the same kind of unstable dynamics due to internal damping forces shown in paper [22] 'On the Stability of Crandall Gyro-pendulum' Physics Letters.

The above-mentioned papers gave us a motivation to see the effects of these nonconservative type forces and its regenerative effects on a general pendulum type rotor and see if one can stabilize the rotors without using heavy dampers or squeeze film actuators with even more smart and elegant control strategies. Paper [5] Rosenberg R.C. and Karnopp D., "Introduction to physical System Dynamics", Mc. Graw Hill, helped us to understand dynamics of physical system actuated with nonpotential fields it also gave us the mathematical insight of forces which are essentially non-potential in nature and how they affect the rotor dynamics and even addressed issues of the instability conditions in a hydrodynamic bearing system. One paper [9] "Theoretical and Experimental Studies on Squeeze Film Stabilizer for Flexible Rotor-Bearing Systems Using Newtonian and Viscoelastic Lubricants" Jr. of Vibration and Acoustics, Transaction of ASME, used squeeze film dampers essentially for large size rotor. Some state feedback control strategies for stabilization of extremely large size rotors was studied [14], [15] but most of the control techniques were using predominantly large number of states as feedback therefore increasing the number of sensors which adds to further complexity in the system. "Modeling and Simulation of Process Machine Interaction in Grinding systems" [23] this paper gave an overall insight that how dry friction forces in between the rotor tool and the mating surface created a tool hunting and wandering effect within the rotor system however no real control strategy to overcome such instability was discussed. $\mathrm{PhD}$ thesis [24] was referred to understand the effects of rotor imbalance and external disturbances in the rotor system and some adaptive control strategies were also studied.

\section{Rotor Instability}

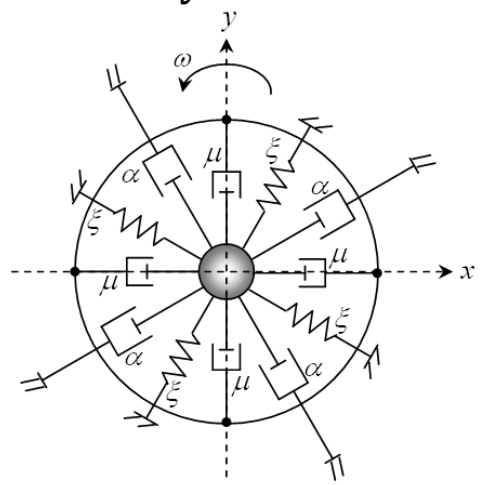

Figure 1: Schematic representation of fixed and rotational dampers

One might choose the overall rotor parameters like the internal damping in the rotating frame, as such that its effective value in all directions is ' $\mathrm{Ri}$ '.

The external damping of the system is taken as such that the effective damping coefficient in all direction is ' $R a$ '. The stiffness coefficient, such that ' $\mathrm{Ks}$ ' is experienced in all directions. If the overall dynamics of the rotor is considered, then the dynamic equation can be given as below

$$
m\left[\begin{array}{c}
\ddot{X} \\
\ddot{Y}
\end{array}\right]=-K\left[\begin{array}{l}
\mathrm{X} \\
\mathrm{Y}
\end{array}\right]-\left[\begin{array}{l}
\dot{\mathrm{X}} \\
\dot{\mathrm{Y}}
\end{array}\right] \cdot \mathrm{R}_{1}+\left[\begin{array}{cc}
0 & \omega \mathrm{R}_{1} \\
-\omega \mathrm{R}_{1} & 0
\end{array}\right]\left[\begin{array}{l}
\mathrm{X} \\
\mathrm{Y}
\end{array}\right]
$$

The above equation can also be written by the overall force components experienced by the rotor in ' $\mathrm{X}$ ' and ' $\mathrm{Y}$ ' directions of the shaft the governing equation in fixed reference frame can be seen from the equation below,

$$
\left[\begin{array}{c}
F x \\
F y
\end{array}\right]=-K\left[\begin{array}{c}
X_{f} \\
Y_{f}
\end{array}\right]+R_{1}\left[\begin{array}{c}
\dot{X}_{f} \\
\dot{Y}_{f}
\end{array}\right]+\left[\begin{array}{cc}
0 & -\omega R_{1} \\
\omega R_{1} & 0
\end{array}\right]\left[\begin{array}{c}
X_{f} \\
Y_{f}
\end{array}\right]
$$

\section{State Space Model:}

$\ddot{o}+\frac{R i}{m} \dot{o}-\frac{(\omega R i-k)}{m} o=0$

$\left[\begin{array}{l}\dot{o}_{1} \\ \dot{o}_{2}\end{array}\right]=\left[\begin{array}{cc}0 & 1 \\ \frac{(\omega R i-k)}{m} & -\frac{R i}{m}\end{array}\right]\left[\begin{array}{l}o_{1} \\ o_{2}\end{array}\right]+\left[\begin{array}{l}0 \\ 1\end{array}\right] u$

Then the dynamic model before the state augmentation can be represented as, $\dot{x}(t)=A x+$ $B u(t), y=H x$, and $x_{0}\left(t_{0}\right)=x_{0}$,

The first augmented state to realize the internal controller may be represented as, $\dot{X}_{\text {ref }}(t)=N r-$ $y=N r-H x$,

That is, $\dot{X}_{\Sigma}=A_{\Sigma} X_{\Sigma}+B_{\Sigma} u+N_{\Sigma} r$,

where $X_{\Sigma}=\left[\begin{array}{c}x(t) \\ x_{\text {ref }}(t)\end{array}\right] \in R^{c}$

Then the augmented state vector will be, $A_{\Sigma}=\left[\begin{array}{cc}A & 0 \\ -H & 0\end{array}\right] \in R^{c \times c}, B_{\Sigma}=\left[\begin{array}{l}B \\ 0\end{array}\right] \in R^{c \times m}$, $N_{\Sigma}=\left[\begin{array}{c}0 \\ N\end{array}\right] \in R^{c \times b}$ 


$$
\left[\begin{array}{c}
\dot{e}_{1} \\
\dot{\mathrm{e}}_{2} \\
\dot{\mathrm{e}}_{3}
\end{array}\right]=\left[\begin{array}{ccc}
0 & 1 & 0 \\
\frac{(\omega R i-k)}{m} & -\frac{R i}{m} & 0 \\
1 & 0 & 0
\end{array}\right]\left[\begin{array}{l}
e_{1} \\
\mathrm{e}_{2} \\
\mathrm{e}_{3}
\end{array}\right]+\left[\begin{array}{l}
0 \\
1 \\
0
\end{array}\right]
$$

The dynamic force components can be seen as simple stiffness dependent forces, ordinary damping force proportional to $\dot{X}_{f}$ and $\dot{\mathrm{Y}}_{\mathrm{f}}$ and lastly the last component of the force which is of special significance and its inherent nature may be discussed. This component of the force can also be called as the regenerative force or the circulating force which exhibits a special feature that it is solely responsible for creating instability in the rotor system due to its anti-symmetric structure.

\section{Instability:}

One might take this peculiar asymmetric force component given below for further analysis.

$$
\begin{array}{r}
{\left[\begin{array}{l}
\mathrm{F}_{\mathrm{xc}} \\
\mathrm{F}_{\mathrm{yc}}
\end{array}\right]=\left[\begin{array}{cc}
0 & -\omega \mathrm{R}_{1} \\
\omega \mathrm{R}_{1} & 0
\end{array}\right]\left[\begin{array}{l}
\mathrm{X} \\
\mathrm{Y}
\end{array}\right] \quad \begin{array}{l}
\text { Therefore, the net work } \\
\text { orbit will be given as, }
\end{array}} \\
\mathrm{W}_{\mathrm{c}}=\oint \overline{\mathrm{F}}_{\mathrm{c} \cdot} \mathrm{dr}=\iint \overline{\mathrm{V}} \times \overline{\mathrm{F}}_{\mathrm{c}} \cdot \mathrm{da}=\iint\left(2 \omega \mathrm{R}_{\mathrm{i}} \hat{\mathrm{k}}\right) \cdot(\mathrm{da}) \hat{\mathrm{k}}=2 \omega \mathrm{R}_{\mathrm{i}} \mathrm{A}
\end{array}
$$

\section{Proposed Smart Embedded Structure and General Features of the Micro- Rotor}

If one considers the basic structure of the rotor from the figure below then the rotor rotates along ' $z$ ' axis

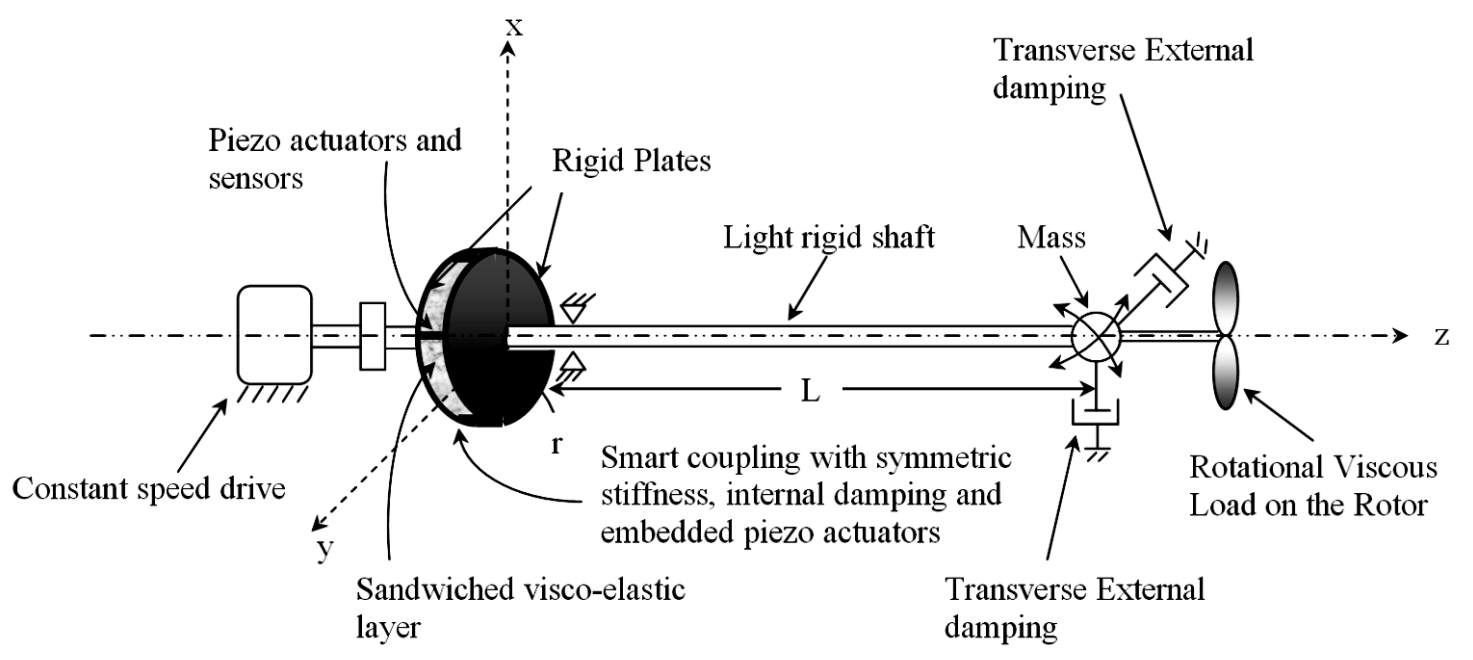

Figure2: Proposed Pendulum like Rotor with Smart Coupling

The integral part of the design includes a smart structure with attached sensor, controller and piezo type actuators within a single coupling. The essential feature of the smart coupling is that it rotates along with the rotor.
The special characteristics of this circulating force component is that it cannot be actually derived from any potential components. One may then say that like most of the other force components found in nature this force is apparently non-potential $\bar{F}_{c} \neq$ $-\bar{V} \Phi$ where the function $\phi(\mathrm{x}, \mathrm{y})$ can be defined as some potential function.

Due to the nature of the circulating force component one might also get a non-vanishing curl from the said dynamics,

$$
\bar{V} \times \bar{F}_{c}=\left(\frac{\partial}{\partial x_{i}} \hat{\imath}+\frac{\partial}{\partial y_{j}} \hat{\jmath}\right) \times\left(-\omega R_{i} y \hat{\imath}+\omega R_{i} x \hat{\jmath}\right)=2 \omega R_{i} \hat{k}
$$

This has a very important and a significant property as because the work done by the circulating component of this force ' $F_{c}$ ' will be path dependent.

We can also say that the network will be done if the point of applications traces a closed orbit. Therefore, the net work done in tracing a closed orbit will be given as,

and it has flexibilities in both ' $\mathrm{X}$ ' and ' $\mathrm{Y}$ ' directions moreover the external damping is attached with the suspension system of the rotor. Both the isotropic stiffness and the internal damping has been taken as a lumped parameter coupled with the smart coupling for ease in modeling the system. 


\section{Controlling the Rotor Shaft by Creating a Negative Damping Coefficient}

(a) Critical or threshold spinning speed calculation and describing the stabilizing methods using translation control law

For the design of a suitable control it is obvious that the running speed ' $\omega$ ' of the rotor should be less than the threshold or the critical spinning speed ' $\omega t h$ '. At speeds greater than the threshold speed of the rotor the regenerative power becomes greater than the dissipative power and the rotor gets destabilized by consuming unwanted power from the constant speed drive motor thus there will be continuous growth of the power drawn by the shaft which actually renders it unstable. This is a highly unstable condition when the power drawn by the shaft increases monotonically. To design a suitable control strategy by making the running speed of the rotor not to cross the threshold speed one may calculate the relationship for the threshold critical spinning speed of the rotor. The smartest way to calculate the critical or threshold spinning speed of the rotor is to compute the regenerative power and the dissipative power and equate the two to address the verge of instability condition. This equivalence of the regenerative and the dissipative power drawn by the shaft helps us to calculate the critical or threshold spinning speed of the rotor which is essential for the controller design.

The relationship for the critical or the threshold spinning speed of the rotor is given as:

$$
\omega_{t h}=\sqrt{\frac{k}{m}}\left(1+\frac{R_{a}}{R_{i}}\right)
$$

The rotor beyond this spinning speed tends to become unstable. Here the critical speed depends on the shaft natural frequency $\sqrt{\frac{k}{m}}$, external damping 'Ra' and material or rotating damping 'Ri'.

The threshold spinning speed of the rotor plays a definitive key role in designing a suitable control strategy. The prime objective of the controller is now to translate the threshold spinning speed ' $\omega_{t h}$ ' further away from the running speed of the rotor. In order to do that the controller has namely three parameters to choose from the expression of the threshold speed.

One may increase the shaft stiffness by feeding back shaft displacement. This way of modulation by the controller on the shaft stiffness is impractical and moreover there would be limitations up to which the stiffness could be increased in flexible rotors with limited number of actuators.

The design of the controller can be done in such a way such that one may increase the shaft stationary damping ' $\mathrm{Ra}$ ' in order to shift the shaft critical spinning speed way beyond the running speed ' $\omega$ ' of the rotor. But some difficulties will be in this approach too let us say if the range $\omega-\omega_{t h}$ ) (that is the difference of spinning and instability onset speed) is too high then higher feedback gains are to be given and since 'Ra' already lies in the numerator of the critical speed relation one may find it difficult to generate such large scale external damping values by the controller. Fixing a high value of the external damping may pose certain difficulties as higher value of the external damping may increase the size of the actuation system itself.

If the size of the actuator itself becomes larger than the size of the rotor this would imply a serious draw back in the selection of the actuating element and fallback in the overall design. There might be severe constraints and problems to integrate such systems with the rotating shaft. There might also be additional problems arising to implement such systems due to high contact friction and wear in the actuator.

Therefore, for practical reasons one might modulate the internal damping $\mathrm{R}_{1}$ thus one can re write the overall equivalent damping $R_{\text {eqv }}=R_{1}-R_{c}$ where $R_{\text {eqv }}$ is effective value of the rotating damping. Therefore, the relationship for the threshold spinning speed is than given as $\omega_{\text {th }}=\omega_{n}\left(1+\frac{R_{a}}{R_{i}-R_{c}}\right)$ here ' $R_{c}$ ' is artificially created which holds the structure of the control law. One may thus modulate the internal damping ' $R_{1}$ ' of the system by feeding back the forces proportional to the velocities in the rotating frame. The overall internal damping of the system may be reduced.

This will translate ' $\omega t h^{\prime}$. way beyond and stabilization of the rotor may be achieved.

This stabilization strategy stands effective as it can be implemented in frame rotating with the shaft. Smart structures may be deployed along with some customized piezo-actuators. Piezo-actuators can be sealed down and miniaturized depending upon the rotor size and can.

To achieve stability the equation can be written as $R_{1}=R_{1}-R_{c}$ where ' $R_{c}$ ' can be taken as a design parameter which means that ' $R_{c}$ ' takes the structure of the control law. A control strategy is needed to design along ' $\mathrm{R}_{c}$ '. One possible way is to modulate directly the shaft drawn power that is the amount of power drawn by the shaft which renders it into unstable whirl already shown in the previous section. If ' $\mathrm{P}_{\mathrm{s}}$ ' is considered as the shaft drawn power which is the actual power responsible for making it unstable then the control law could be well designed as,

$$
\mathrm{R}_{\mathrm{c}}=\alpha \mathrm{P}_{\mathrm{s}}+\beta \int_{0}^{\mathrm{t}} \mathrm{P}_{\mathrm{s}} \mathrm{dt}+\gamma \frac{\mathrm{dP}_{\mathrm{s}}}{\mathrm{dt}}
$$


The following relation explains how the threshold running speed gets modulated

$$
\omega_{\text {th }}=\omega_{n}\left(1+\frac{R_{a}}{\left(R_{i}-\alpha P_{s}-\beta \int_{0}^{t} P_{s} d t-\gamma \frac{d P_{s}}{d t}\right)}\right)
$$

Some disadvantage of the proposed controller is that it is already a frustrating task to measure the actual shaft drawn power 'Ps'. The disadvantage of directly measuring or sensing 'Ps' is that certain amount of power will be used for running the additional load and also some power will be lost to the environment via external damping therefore to determine the amount of power which actually renders the shaft unstable is a tedious task. Instead of taking the shaft drawn power 'Ps' as the sensing element one may resort to the orbital speed of the rotor itself. This is a good design as because the orbital speed is proportional to the area of the orbit taken by the rotor which is indirectly proportional to the shaft drawn power 'Ps'. As stated earlier that sensing of the shaft drawn power 'Ps' is not practical one may introduce an orbital function mentioned below such that using a singleton PID structure like controller one can achieve stabilization in both ' $\mathrm{X}$ ' and ' $Y$ '. The proposed controller structure reduces the number of controllers as opposed to conventional PID controllers based on errors coefficients in ' $\mathrm{X}$ ' and ' $\mathrm{Y}$ ' displacement.

One may sense the orbital area given as $X_{r}{ }^{2}+Y_{r}^{2}$ but even better response function would be the velocity response function $\dot{X}_{r}{ }^{2}+\dot{Y}_{r}^{2}$ as because the whirl orbiting speed is the natural frequency of the shaft without any damping.

Therefore, the overall control structure could now be given as,

$$
\begin{gathered}
R_{c}^{\prime}=\left(\alpha * V_{\text {amp }}^{2}+\beta * \int_{0}^{t} V_{a m p}^{2} d t+\sigma * d V_{a m p}^{2} / d t\right) \\
\text { where } V^{2}{ }_{a m p}=\dot{X}_{r}^{2}+\dot{Y}_{r}^{2}
\end{gathered}
$$

\section{Fractional Order PID Controller Design for a Extremely Small Size Rotor System Based on Conventional Frequency Methods}

A generalized $\mathrm{PI}^{\lambda} \mathrm{D}^{\mu}$ controller of the form:

$$
\mathrm{C}(\mathrm{s})=\mathrm{K}_{\mathrm{p}}+\mathrm{K}_{\mathrm{i}} / \mathrm{s}^{\lambda}+\mathrm{K}_{\mathrm{d}} \mathrm{S}^{\mu}
$$

Where $\lambda$ and $\mu$ are the fractional orders of the integral and derivative parts of the controller, respectively.
Since this kind of controller has five parameters to tune $\left(K_{p}, K_{d}, K_{i}, \lambda, \mu\right)$, up to five design specifications for the controlled system can be met, that is, two more than in the case of a conventional PID controller, where $\lambda=1$ and $\mu=1$.

(a) Phase margin $\phi \mathrm{m}$ and gain cross-over frequency $w_{\text {cg }}$ specifications.

Gain and phase margins have always served as important measures of the robustness. It is known that the phase margin is related to the damping ratio of the system and therefore can also serve as a performance measure. The equations that defines the phase margin and the crossover frequency are:

$$
\begin{gathered}
|C(j w c g) G(j w c g)|_{d B}=0 d B ; \\
\arg \left(C\left(j w_{c g}\right) G\left(j w_{c g}\right)\right)=-\pi+\phi_{m}
\end{gathered}
$$

(b) Robustness to variations in the gain of the plant.

The next constraint considered is:

$$
\left.\frac{d \arg (F(s))}{d w}\right|_{\mathrm{w}=\mathrm{wcg}}=0
$$

(c) High- frequency noise rejection.

A constraint on the complementary sensitivity function T(jw) can be established:

$$
\begin{aligned}
& \left|T(j w)=\frac{C(j w) G(j w)}{1+C(j w) G(j w)}\right| \mathrm{dB} \leq \mathrm{AdB}, \\
& \forall \mathrm{W} \geq \mathrm{w}_{\mathrm{t}} \mathrm{rad} / \mathrm{sec}=>\left|T\left(j w_{t}\right)\right|=\mathrm{AdB}
\end{aligned}
$$

With " $A$ " the desired noise attenuation for frequencies $\mathrm{w} \geq w_{t} \mathrm{rad} / \mathrm{sec}$.

(d) To ensure a good output disturbance rejection.

A constraint on the sensitivity function $S(j w)$ can be defined as:

$$
\begin{gathered}
\left|S(j w)=\frac{1}{1+C(j w) G(j w)}\right| \mathrm{dB} \leq \mathrm{B} \mathrm{dB}, \\
\forall \mathrm{W} \leq \mathrm{w}_{\mathrm{s}} \mathrm{rad} / \mathrm{sec}=>\left|S\left(j w_{s}\right)\right|=\mathrm{B} \mathrm{dB}
\end{gathered}
$$

With "B" the desired value of the sensitivity function for frequencies $\mathrm{w} \leq \mathrm{w}_{\mathrm{s}} \mathrm{rad} / \mathrm{sec}$ (desired frequency range). After finding out the A, B, C, D matrices the gain parameters $[K p, K i, K d, \mu, \lambda]$. For the following designing specifications: Phase Margin = 60 degree, Gain Margin $=10 \mathrm{~dB}$ Gain crossover frequency $=0.10 \mathrm{rad} / \mathrm{s} \mathrm{A}=-20 \mathrm{~dB}$ at $w$ ? $w t=10 \mathrm{rad} / \mathrm{s}$ $\mathrm{B}=-20 \mathrm{~dB}$ at $t w$ ? $w=.01 \mathrm{rad}$ 


\section{Dynamics without Implementation of Stabilizing Strategy}

The rotor is rotated at a speed beyond. There is no eccentricity applied to the system.

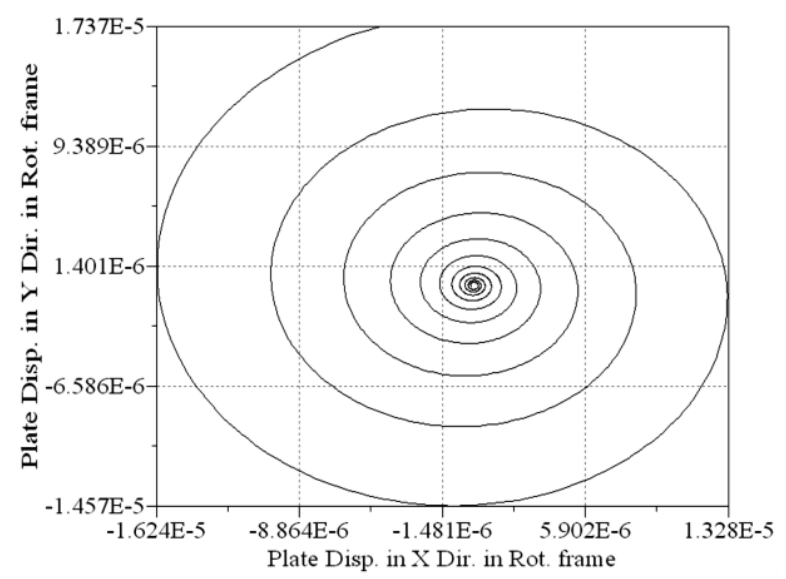

Figure 6: Instability in a simple Rotor without active stabilization

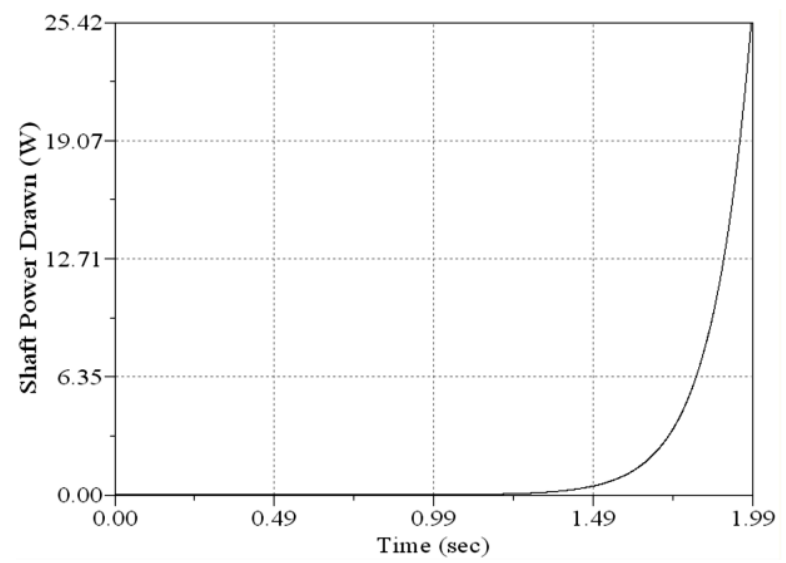

Figure 7: Increasing shaft drawn power which renders the rotor unstable

\section{Dynamics with Implementation of FOPID Controller}

The parameters for this set of simulations are Mass $=0.1 \mathrm{~kg}$, Shaft stiffness $=1000.0 \mathrm{~N} / \mathrm{m}$, Stationary Damping=0.2 Ns/m, Rotating Damping=0.02 Ns/m, Driving Speed=660 rad $/ \mathrm{sec}$.

The fig. 8 illustrates the ' $\mathrm{X}$ ' displacement, which apparently gets stabilized and fig 9 is the Stabilization in ' $\mathrm{Y}$ '. The rotor speed is $660 \mathrm{rad} / \mathrm{s}$ and the stabilizing action lifts the threshold speed to $1127 \mathrm{rad} / \mathrm{s}$. This demonstrates the stabilization effect how the reduction in effective internal damping.

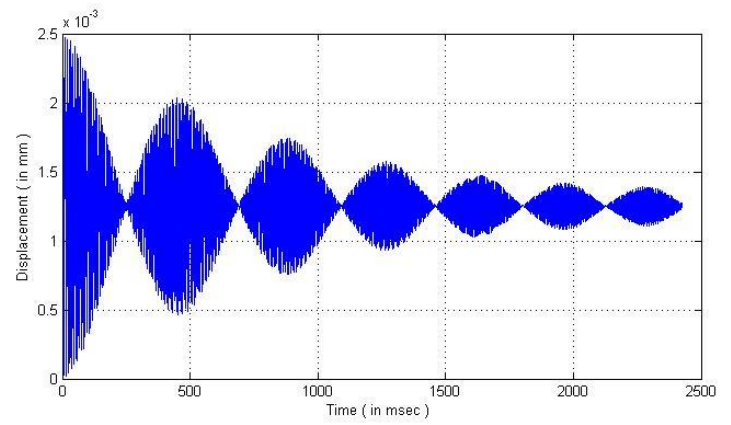

Figure 8: Stabilization of the ' $X$ ' Displacement of the rotor with a FOPID type controller at speed 600RPM

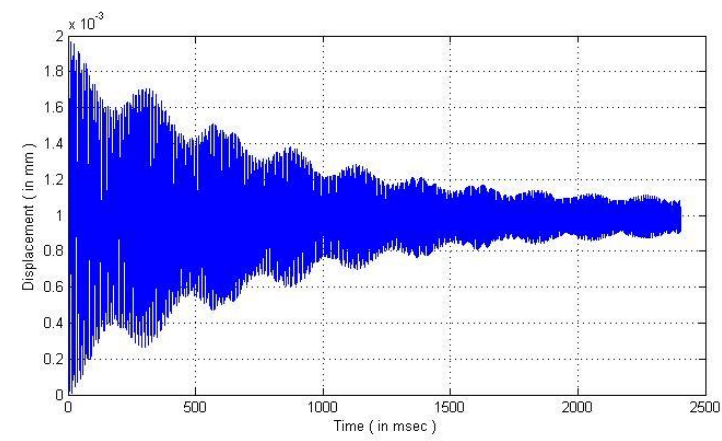

Figure 9: Stabilization of the ' $Y$ ' Displacement of the rotor with a FOPID type controller at 600 RPM

\section{A Tuned Variable Structure Control and its Efficacy to Stabilize Small Size Rotors with Internal States as a Feedback}

Extraction of the sliding surface from geometric transformation model

Given a set of desired poles $\left\{\lambda_{i} ; \quad i=1,2, \ldots \ldots \ldots \ldots . . . n\right\}$ the system can be restricted to a sliding surface following the fundamental steps below.

(a)Initial goal is to check the exi.stence of the transformation matrix $T(x)$.

$z=\tau(x), \quad \tau(0)=0$

For any system model $\dot{x}=f(x)+g(x) u$

One may also check the inverse $x=T^{-1}(x)$.

One may conceive to design fundamentally the polynomial $T(x)$ and check weather transformation exists.

$\dot{z}(t)=\frac{\partial T}{\partial x} \dot{x}=\frac{\partial T}{\partial x}(f(x)+g(x) u)$ 


$$
\left.\frac{\partial T}{\partial x} f(x)\right|_{x=T^{-1}(z)}=\left[\begin{array}{c}
z_{2} \\
z_{3} \\
\vdots \\
z_{n} \\
\bar{f}_{n}\left(z_{1}, z_{2}, \ldots \ldots, z_{n}\right)
\end{array}\right]
$$

and

$$
\left.\frac{\partial T}{\partial x} g(x)\right|_{x=T^{-1}(z)}=\left[\begin{array}{c}
0 \\
0 \\
\vdots \\
0 \\
1
\end{array}\right]
$$

Considering the equation in matrix form, Because $z=\tau(x)$ one may conclude

$\frac{\partial \tau_{i}}{\partial x} f(x)=\tau_{i+1}, \quad$ for $i=1,2, \ldots \ldots, n-1$

Or equivalently

$\tau_{i+1}=\alpha_{f} \tau_{i}, \quad i=1,2, \ldots ., n-1$

Therefore, introducing the Lee derivatives, the final transformation $z=\tau(x)$ has the form

$$
T=\left[\begin{array}{c}
T_{1} \\
L_{f} T_{1} \\
\vdots \\
L_{f}^{n-2} T_{1} \\
L_{f}^{n-1} T_{1}
\end{array}\right]
$$

a) If T exists then $[T, B]$ is invertible.

b) $\left[\begin{array}{ll}T & B\end{array}\right]^{-1}=\left[\begin{array}{l}T^{T} \\ B^{T}\end{array}\right]$

c) One may find matrix $f \in R^{m \times(n-m)}$ such that eig $\left(T^{T} A T-T^{T} A B F\right)=\left(\lambda_{1}, \lambda_{2}, \ldots \ldots ., \lambda_{n-m}\right)$

d) Matrix $W=(T-B F)$

e) Final computation of the sliding surface polynomial the last ' $m$ ' number of rows of $\left[\begin{array}{ll}W & B\end{array}\right]^{-1}$ could be used for the effective sliding surface $S x=0$.

\section{* Design of the controller in the sense of Lyapunov}

A variable structure control could actually use the Lyapunov's criteria in determining the control command $u(x, t)$ that may actually drive the state trajectory to the equilibrium manifold.

Choosing a quadratic function for the controller

$V(t, x, \sigma)=\sigma^{T}(x, t) W \sigma(x, t)$,

One may consider ' $W$ ' as a symmetric positive definite matrix.
To finally design the controller one may take the time derivative of $V(t, x, \sigma)$ negative definite for $\sigma \neq \mathbf{O}$.

Taking the derivative of $V(t, x, \sigma)$ one gets

$\dot{V}(t, x, \sigma)=\dot{\sigma}^{T} W \sigma+\sigma^{T} W \dot{\sigma}=2 \sigma^{T} W \dot{\sigma}$

$\dot{V}=2 \sigma^{T} W \frac{\partial \sigma}{\partial t}+2 \sigma^{T} W \frac{\partial \sigma}{\partial x} f+2 \sigma^{T} W \frac{\partial \sigma}{\partial x} B u<0$

For $\sigma \neq \mathrm{O}$, i.e. $\dot{V}(t, x, \sigma)$ is negative definite. the combined control structure could be taken as $u(x, t)=u_{e q}(x, t)+u_{N}(x, t)$

Taking,

$\dot{V}(t, x, \sigma)=2 \sigma^{T} W \frac{\partial \sigma}{\partial x} B(x, t) u_{N}(x, t)<0$

If $\frac{\partial \sigma}{\partial x} B(x, t)$ is assumed to be non-singular for all ' $t$ ' and ' $x$ ' it is then possible to take the control law

$u_{N}(x, t)=\left[\frac{\partial \sigma}{\partial x} B(x, t)\right]^{-1} u_{N}(x, t)$

for the given problem switching control with state dependent gains are used whose control law may take the form

$u_{N_{i}}=\alpha_{i i}(x, t) \operatorname{sgn}\left(\sigma_{i}(x, t)\right)$,

The condition for stability can be taken as

$=2 \alpha_{i i}(x, t)\left|\sigma_{i}\right|=0$

for $\sigma_{i} \neq \mathbf{O}$.

Where,

$\mathrm{k}=$ spring constant $=1000$

$\mathrm{m}=\mathrm{mass}=0.1$

$\mathrm{W}=$ angular frequency $=660$

$\mathrm{Ri}=$ internal damping $=0.02$

$\sigma=$ sigma

$\alpha=$ alpha

And here,

$$
\begin{gathered}
X=X_{1}, Y=Y_{1} \\
X^{\prime}=X_{2}, Y^{\prime}=Y_{2} \\
\sigma=74.4 X_{1}-2.05 X_{2}-2.93 Y_{1}+3.93 Y_{2} \\
\alpha=\frac{+0.02 \times 2.05) X^{\prime}-\left(2.05 \times 10^{4}-3.93 \times 132\right) X+(74.41}{3.93 Y^{\prime}-2.05 X^{\prime}}
\end{gathered}
$$

The sliding surface is $u=\alpha \operatorname{sat}(\epsilon)$

Where $\epsilon=$ constant $=0.000001$

The control logic is

$\sigma<0=\left\{\begin{array}{l}u=\alpha+\epsilon \\ u=\alpha-\varepsilon\end{array}\right.$

$\sigma>0=\left\{\begin{array}{l}u=\alpha+\epsilon \\ u=\alpha-\varepsilon\end{array}\right.$ 


\section{Stabilization Results with Tuned Variable Structure Control}

The parameters for this set of simulations are Mass $=0.1 \mathrm{~kg}$, Shaft stiffness $=1000.0 \mathrm{~N} / \mathrm{m}$, Stationary Damping=0.2 Ns/m, Rotating Damping=0.02 Ns/m, Driving Speed=660 $\mathrm{rad} / \mathrm{sec}$.
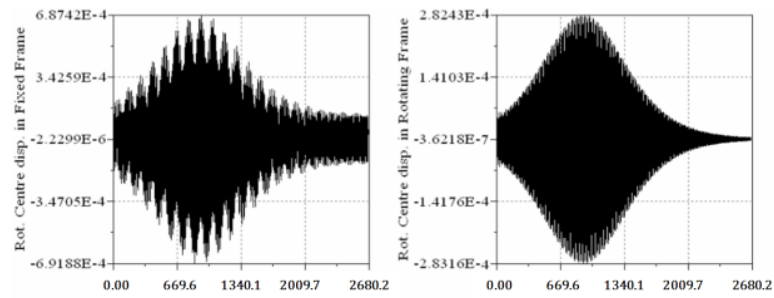

$\tau \quad$ (a) (b)
Figure 10: Sliding mode control of the Rotating shaft Results and Discussions

Tabele 1. Comparative Table between FOPID and simple Sliding mode control.

\begin{tabular}{|c|c|c|}
\hline $\begin{array}{c}\text { Controller } \\
\text { Comparison }\end{array}$ & $\begin{array}{l}\text { Maximum } \\
\text { displacement } \\
\text { (in } \mathbf{~ m m} \text { ) }\end{array}$ & $\begin{array}{c}\text { Final comment } \\
\text { on the control } \\
\text { strategy }\end{array}$ \\
\hline $\begin{array}{l}\text { FOPID } \\
\text { (Tuned) }\end{array}$ & $\begin{array}{c}\mathrm{X}(\text { displacement })= \\
25 \times 10^{-3} \mathrm{~mm}, \\
\text { And Y } \\
(\text { displacement })= \\
2 \times 10^{-3} \mathrm{~mm}\end{array}$ & $\begin{array}{l}\text { Reduced in the } \\
\text { order- } 10^{-3} \mathrm{~mm}\end{array}$ \\
\hline $\begin{array}{l}\text { Sliding } \\
\text { mode } \\
\text { (Tuned) }\end{array}$ & $\begin{array}{c}\mathrm{X}(\text { displacement })= \\
6.8 \times 10^{-4} \mathrm{~mm}, \\
\text { And Y } \\
(\text { displacement })= \\
2.82 \times 10^{-4} \mathrm{~mm}\end{array}$ & $\begin{array}{l}\text { Comparatively } \\
\text { Better control } \\
\text { action in the } \\
\text { order- } 10^{-4} \mathrm{~mm}\end{array}$ \\
\hline
\end{tabular}

One may see that initially the structure of the controller was taken like a fractional FOPID with internal states having two independent directions in ' $\mathrm{X}$ ' and ' $\mathrm{Y}$ ' the self tuning resulted in a stabilized state up to $10^{-3} \mathrm{~mm}$ in convergence but still the controller worked quite efficiently to stabilize rotors of such extreme diameters and finally a well tuned sliding mode controller could even improve the damping level to up to $10^{-4} \mathrm{~mm}$ this is a e good result considering rotors of this extreme size.

\section{Conclusion}

Instability issues for large size rotors due to internal damping or material damping of the rotor material is quite well known. Control strategies to stabilize such large size rotors with large mechanical structures and dampers are common but highly inefficient due to overhaul cost, maintenance and overall complexity of the system. Here in the paper firstly instability analysis of rotors in general is discussed due to material damping and later an elegant Tuned FOPID algorithm is developed which is applicable for stabilizing relatively small mini or micro size rotor.

One may find the dynamics of these coupled systems highly challenging therefore for simplicity in control structure the orbital velocity state of the rotor was taken as the principle feedback sate within a FOPID structure. This way of stabilization was found efficient.

A well-tuned sliding mode controller raised the damping levels of the system such that it gave grossly a much better stabilization in terms of both convergence. It was observed that the convergence of a tuned variable structure control stabilized the ' $\mathrm{X}$ ' and ' $Y$ ' displacement of the rotor in the order of $10^{-4}$ $\mathrm{mm}$ compared to a conventional FOPID like controller.

\section{References}

[1] MeirovitchL., "Analytical methods in vibration", N.Y Macmillan, 1967

[2] Crandall S.H. Karnopp, D.C. and Kurtz D.C., "Dynamics of Mechanical and electromechanical systems", McGraw Hill, 1968

[3] Holmes R., "Nonlinear Performance of Squeeze Film Bearings" Jr. of Mechanical Engineering Science. 1972, 14(1), 74-77.

[4] Harnoy, A "An Analysis of Stress relaxation in Elastico-Viscous Hydrodynamic Lubrication of Sleeve Bearings" ASLE Transactions, 1976, Vol.19, pp301

[5] Rosenberg R.C. and Karnopp D., "Introduction to physical System Dynamics", Mc. Graw Hill, NewYork,1983

[6] Mukherjee A., "Effect of Bi-phase Lubricants on dynamics of Rigid Rotors" Transaction of ASME, Jr. of Lubrication Technology, 1984, 105, pp 2

[7] BazA. and Poh S., "Performance of an active Control System with Piezo electricactuators", Journal of sound and Vibration, 126(2) , p.327324, 1988

[8] Margolis D. L., Karnopp D. C. and Rosenberg C. R., "System Dynamics a unified approach" John wiley and sons, 1990

[9] Halder B., Mukherjee A. and Karmakar R., "Theoretical and Experimental Studies on Squeeze Film Stabilizer for Flexible RotorBearing Systems Using Newtonian and Viscoelastic Lubricants" Jr. of Vibration and Acoustics, Transaction of ASME, 1990, 112(4), 473-482.

[10] Burdess J.S. and Fawcett J.N., "Experimental evaluation of a piezoelectric actuator for the control, of vibration in a cantilever beam", IMECHE, Part I: J. Sys. and control eng., Vol. 206, 1992, p99-106

[11] Preumont A., DufourJ. and Malekian C., "Active Damping by a Local force Feed Back With Piezo 
Actuatotrs", Journal of Guidence and control., VOL 15No.2, March April 1992, pp.390-395.

[12] Smith R.D. and Weldon W.F., "Nonlinear control of a rigid rotor magnetic bearing system modeling and simulation with full state feedback" IEEE transaction on Magnetics vol. 31 NO2 March 1995

[13] Vaz J. A. C., "Theoretical and Experimental Studies on the Dynamics and Control of Intelligent Beam Structure with Special Reference to Flexible Manipulators" PhD Dissertation Department of Mechanical Engineering, Indian Institute of Technology, 1995 Kharagpur, Pin 721 302, India.

[14] Bentley J.P., "Principle of Measurement system" Third edition, 1995, Pearson

[15] Murthy D.V.S., "Transducer and Instrumentation" PHI, 2003

[16] Ballas R. G., "Piezoelectric Multilayer Beam Bending Actuators" Springer, 2006, ISBN 978-3540-32641-

[17] Mukherjee A., Karmakar R. and Samantaray A. K., "Bond Graphs in Modeling, Simulation and Fault Identification". 2006, I. K. International Publishing House Pvt. Ltd. India, CRC Taylor and Francis, USA.

[18] Samantaray A. K., Bhattacharyya R. and Mukherjee A., "An Investigation in to the Physics Behind the Stabilizing Effect of Two Phase Lubricants in Journal Bearings" Jr. of Vibration and Control, 200612,(1), 425-442.

[19] Kirillov O. N., "Gyroscopic Stabilization in the Presence of Non-conservative forces" Dokl. Math, 2007, 76, 780-785.
[20] Glebov V. V., Sorokin V. M., Puchkov V.P. and Ignat'ev D. A., "Eliminating Vibrations in the Face Grinding of Glass" Russian Engineering Research, 200727, 305-308.

[21] Bou-Rabee N. M., Marsden J. E. and Romero L. A., "Dissipation-Induced Heteroclinic Orbits in Tippe Tops"2008, SIAM Review, 50(2), pp 325344.

[22] SamantarayA. K., Bhattacharyya R. and Mukherjee A., "On Stability of Crandall Gyropendulum" Physics Letters, 2008A 372,238243.

[23] Aurich A., Bierman D., Blum H., Brecher C., Carstensen C., Denkena B., Kloche F., Kroeger M., Steinmann P. and Weiner K., "Modelling and Simulation of Process Machine Interaction in Grinding" Production Engineering Research and Development. 3, 2009111-120.

[24] Simon A., "Adaptive Disturbance Rejection and stabilization for rotor systems with internal damping", Phd. dissertation graduate faculty of Auborn University 2009.

[25] Ortega A.B., Carbajal F.B., Navarro G.S. and Salazar M.A.O., "Active vibration control of the rotor bearing system based on dynamic stiffness" Rev facts Aniv

[26] Active stabilization of rotors with circulating forces due to spinning dissipation Journal of Vibration and Control September 2011 17: 1509-1524, November 22, 2010

[27] A proposal for active control of face abrasive tool wandering Journal of Vibration and Control1077546311402708, first published on May 20, 2011 